\title{
A Chain Oriented Data Collection Protocol for Energy-Aware and Delay-Constrained WSN
}

\author{
Nahdia Tabassum ${ }^{\dagger}$, Quazi Ehsanul Kabir Mamun ${ }^{\dagger}$, A K M Ahsanul Haque ${ }^{\dagger \dagger}$, Yoshiyori Urano ${ }^{\dagger}$ \\ GITS, Waseda University, Tokyo 169-0051, Japan \\ ${ }^{\dagger}$ ECE Discipline, Khulna University, Khulna 9208, Bangladesh \\ †\{nahdia@akane., mamun@fuji., urano@\}waseda.jp, ${ }^{\dagger}$ ahsanku@gmail.com
}

\begin{abstract}
Energy awareness plays an important role in developing routing protocol for the battery powered wireless sensor networks. As the replacement of the battery is often unfeasible in practical situations, we present here an optimal solution for the maximum utilization of precious available energy at the same time trying to minimize the latency in data delivery. We propose to form hierarchical chains with deployed sensors to collect information from a target field where data get fused at every node level before transmitted finally. Our protocol utilizes the higher energy nodes for more frequent long distance transmissions so that the energy expenditure become even between all nodes in the network irrespective of their physical locations. It has been found in our simulation that this protocol outperforms other hierarchical protocols like LEACH and PEGASIS in both the cases of energy consumption and time requirements respectively. It has been also found that the overall lifetime of the sensor network also increases in our protocol.
\end{abstract}

Keywords- Sensor network, chain based protocol, energyawareness, data collection, routing and latency.

\section{INTRODUCTION}

Unlike the traditional wireless networks, sensor networks are characterized as application dependent network due to the fact that in different situations they are intended to carry out different type of jobs depending on the nature of the specific applications $[1$, $2,3]$. But in almost all cases the principle nature of work for sensors is to collect information from a target field, process the information, and send them for further processing to some sink or base station (BS) which may be located inside or at far from the sensor field [4]. There are wide ranges of application areas for sensor networks which includes complex and hazardous environment monitoring, simple habitat monitoring, critical military applications, disaster management, medical condition monitoring and so on $[1,5,6]$. Most of these applications require periodical delivery of data from the target field with low duty cycle. But some of them would require this data gathering process can be accomplished with very low latency during each duty cycle, e.g. in security or battlefield surveillance.

The latest state of the art advances in micro-electromechanical (MEMS) systems, low-power analog and digital electronics, have enabled us to produce low-cost sensor devices which set an opportunity to densely deploy these tiny devices in an ad hoc manner to collect desired information from inaccessible terrain in an autonomous manner $[7,8,9]$. However, there are some criteria that the sensor networks should meet in order to come in reality. First of all energy scarcity is the most crucial parameter that should be addressed in designing a communication protocol of these distributed, self organized networks because in most of the cases it is impossible to replace the battery once they are drained out for thousands of nodes in a harsh and inaccessible terrain [4]. The other issues those need to be taken care of include data aggregation, fault tolerance, latency in data delivery, self-organization, security etc. $[10,11]$.

It is unlikely that one particular protocol for wireless sensor networks can satisfy all possible application scenarios. Obviously network protocols vary according to the specific nature of the application [3]. In our experiment we consider a periodical data collection problem from a remote target field in both energy and time efficient manner. Here we consider that the sensor network consists of a hundred of randomly deployed static nodes with the BS located at a far position from the network field. All the nodes continuously sense and transmit this sensed data in a systematic and periodic way. Hence all the nodes are similar in capacity that is they have equal computing, processing and communication capabilities. We also consider that the BS has no energy lacking and capable of reaching each node directly but sensor nodes though capable of reaching the BS directly, are severely energy constrained.

In this paper we give an extended explanation and simulation results of our work described in [12, 13], where we proposed an improved energy efficient hierarchical periodical data collection protocol for 
wireless sensor networks. The issues that we particularly addressed in our work are, how to route the sensed data from all the nodes in an energy efficient manner so that the lifetime of the network is maximized and how to reduce the delay in data delivery to BS. We took the advantage of data aggregation as it reduces the cost of transmission a lot while transmitting aggregated information from node to node than to transmit raw data $[14,15,16]$. Hence we propose a Chain Oriented SEnsor Network (COSEN) protocol which is a two-layer protocol where a number of chains are formed in order to cover the whole region. At first several small, fixed-length chains are constructed using a greedy algorithm and a chain leader is selected in each chain based on several criteria. These chain leaders again form a higher-level chain and among those leaders one is chosen depending on some considerations to accumulate all data from the network and send that data to the remote BS at each round whereas other nodes only communicate with its neighbor in the chain. Chain leaders as well as higher-level chains changes after few rounds and higher-level leaders may change at every round. Due to the small length of data collection chain and small communication distances between nodes, time required for data collection as well as energy required in routing data decrease significantly. Beside these in our proposal data get fused at every node level when transmitted from one node to another and finally reach at chain leader. Data also get fused while routed among higher-level chain until it reaches the higherlevel leader and finally transmitted to the BS.

The remainder of this paper is organized as follows. Section II presents an overview of the related works. We discuss in details about the protocol architecture in section III. Section IV discusses about simulation environment and various simulation results. Finally our protocol's achievements are shown in section V. A brief conclusion and future work are stated in section VI.

\section{RELATED WORKS}

Now we are aware of the fact that energy consumption is a key issue that needs to be addressed at every level of network layer for a wireless sensor network. Researchers have invested their thoughts and came out with some very good solutions in this regard. Most of the protocols can be classified as either datacentric or hierarchical or location-based [14]. Datacentric routing protocols mainly utilizes attribute-based query and involve naming of desired data. In a hierarchical routing protocol, the network is divided into clusters with one node acting as local cluster leader in each cluster. Lastly, the location-based routing protocols deal with node's position to route data towards desired location. It is found in literature that while considering energy awareness and time complexity for periodic data collection applications, hierarchical routing protocols perform better in comparison with their kind of other solutions [17]. To name a few are Low Energy Adaptive Clustering Hierarchy (LEACH) [18], Power Efficient Gathering in Sensor Information Systems (PEGASIS) [19], Threshold sensitive Energy Efficient sensor Network protocol (TEEN) [20], and Base station Controlled Dynamic Clustering Protocol (BCDCP) [17].

Our work is inspired by hierarchy based protocol LEACH and chain based protocol PEGASIS. We took up both of their novel views and tried to minimize the shortcomings of those.

LEACH is a protocol that considers clusters in a homogeneous sensor field with nodes with two roles. One is to work as higher-level node or cluster head with responsibility to accumulate data from the members of its cluster, aggregate them to a single unit of information and finally transmit that to remote BS. Being a leader, nodes need more transmission and processing to do, those eventually cause faster power drain in these nodes. So LEACH proposes rotation of the role as cluster head among all nodes at every round. This is to achieve power dissipation balancing among the nodes and thus to help the network run longer. LEACH is suitable for networks where every node has data to send at regular intervals. The beauty of this protocol is, it eliminates many direct long distance transmissions and reduces amount of data by implementing data aggregation at header level of nodes as compared to direct transmission. But still there are quite a good number of long distance transmissions for data those are transmitted directly from each cluster head to the remote BS. Here, the cluster setup overload that needs to be carried by the network at every round asks for considerable energy dissipation. Also, due to random cluster setup and single hop communication between nodes to cluster heads, there is a certain possibility that the distances between them are not short enough to keep transmission energy at its minimum.

On the other hand PEGASIS addresses this problem with a chain based solution. Authors tried to minimize the number of long distance transmissions from cluster heads to BS and looked for minimum distance transmissions. To satisfy the minimum transmission distance it forms a chain of all the nodes in sensor field. Only one member of the chain takes the responsibility to transfer the aggregated information to BS. Selection of this node is in random so that energy consumption is shared among all nodes. It does not propose any dynamic cluster formation thus any sort of overload associated with this at every round are also avoided. The strength of this view is, it proposes a 
simple greedy algorithm to find the next node of the chain, which leads to faster network setup time and easy implementation of the protocol. PEGASIS identifies its closest neighbor by sending a power signal to its neighbor nodes and gradually reducing the power signal till it finds the nearest node. Also data aggregation takes place while a node transfers to the next member in the chain. It shows somewhat better performance in case of overall energy consumption of network as it avoids long distance transformations but lacks while data delivery time is a concern. Due to a single chain, time required to collect data from the end node of the chain to the leader node yields large. Sometime this delay can be too much for time sensitive applications if the network consists of large number of nodes.

TEEN is another modification of LEACH, which provides responses to drastic and sudden changes in the network. But because of its reactive nature it is not always suitable for periodic data collection scenario.

BCDCP also provides an improvement over LEACH where the energy expensive works like cluster setup or routing path calculations etc. are carried out by the BS, which has no energy limitations as network nodes.

In our case, we tried to provide an alternative solution without involving BS thus our proposal is completely self-organized and energy efficient with very limited delay. Cluster setup rounds are avoided so as its associated overheads. Chain length is also shortened to avoid excessive delay. It can find applications in periodical data collection environments such as battlefield surveillance or industrial monitoring. The proposed protocol achieves almost equal energy efficiency as of PEGASIS but with much less delay.

\section{Protocol ARChitecture Details}

Architecture of our protocol can be described in two steps. Firstly we describe in details about the chain formation phase where several chains called lowerlevel chains are formed including all the live nodes in the network and then a lower-level leader is selected in each chain based on some criteria. These lower-level leaders in turn form a higher-level chain from which a single leader called higher-level leader is finally selected depending on some parameters to transmit to the BS. Other issues like how many nodes should be included in each chain, after how many rounds lowerlevel leaders should be changed and after how many rounds chain reconstruction should take place are discussed in the following section. Secondly we will discuss about the data collection and transmission phase that includes the node level data collection, data

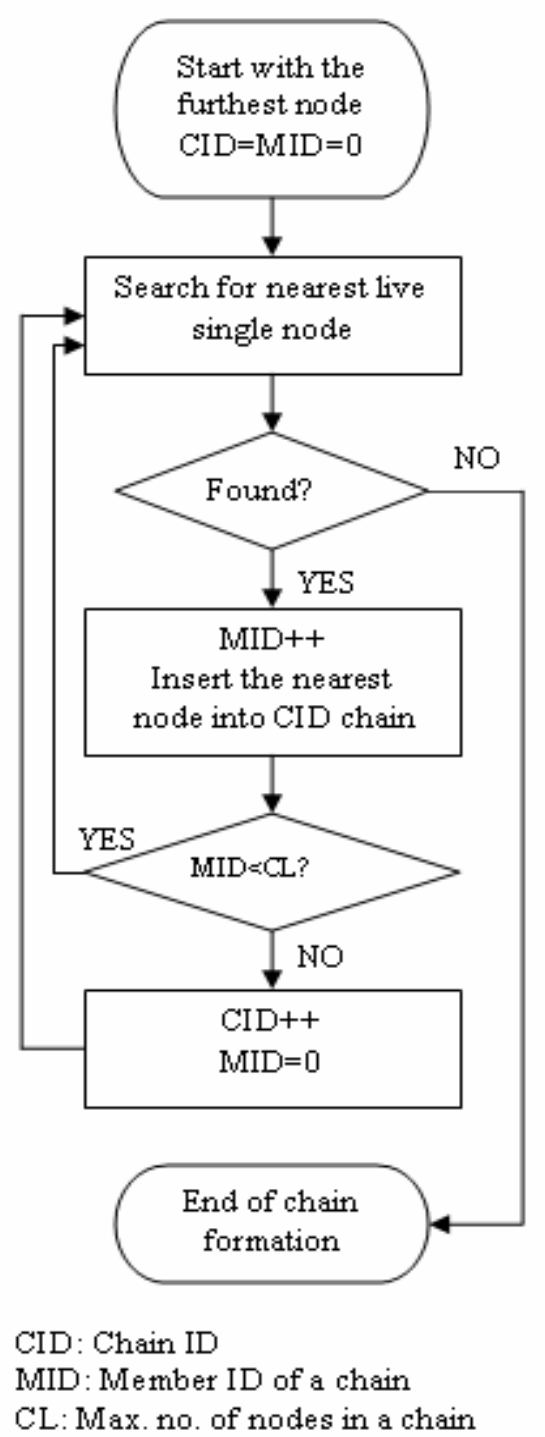

Figure 1. Flowchart of chain formation

aggregation and the final transmission of information to remote BS.

In designing the protocol we took several assumptions. It is assumed that nodes are aware of their positions. In the chain formation phase, localization knowledge would be beneficiary for us as chain formation starts from the furthest node in the network. By using a greedy algorithm we include each nearest node within a chain. It has been observed that inter-node distance gradually increases in the chains particularly in the last chain because nodes once included in any chain cannot be included again. Therefore the main point of forming chains from furthest position benefits us smaller inter-node distances for the nodes which are located far from the BS. As these nodes require more energy than the nodes in the nearer positions to transmit to the BS, it will 
definitely make a balance in energy expenditure throughout the network. Positions of the nodes may be obtained by methods based on triangulation $[10,21]$, where nodes approximate their position using radio strengths from a few known points. N. Bulusu et al. in [21] showed that algorithms based on triangulation or multilateration can work quite well under conditions where only very few nodes know their positions a priori. Certainly this extra negotiation consumes extra energy but as our network is considered as static one, this process may take place only once at the beginning of network setup and can be considered negligible.

\section{A. Chain Formation Phase}

During the chain formation phase node at the furthest position from BS takes initiative to start the process. We also assumed that sensor nodes have dynamic power adjustment capability so that while transmitting, they can modify the amplifier power in such a way that it can be heard only by the closest neighbor in the chain. This ensures the avoidance of collision in large extent. Figure 1 shows the flowchart of the chain formation algorithm where chain formation starts from the furthest node in the network that gives itself the initial chain and member id. Then it finds out the next alive node that is not included in any other chain. The next node's chain id is same as previous but member id is incremented by one. In this way chain formation continues until the member id reaches some maximum number. In our present experiment we took this maximum number to be 20 . This choice of maximum number of nodes in a chain depends on several factors such as energy consumption, tolerable delay etc. those we discuss more elaborately in sub-section IV.B. Whenever member id in a chain reaches its maximum number, the next node increments its chain id by one and assigns itself the initial member id for that chain. This way chain formation continues until all the live sensors in the network are included in several chains. These are the chains which we refer as lower-level chains.

These lower-level chains remain fixed for long duration of time and chain formation takes place again whenever $20 \%$ of member nodes of the previous chain formation die. This is to maintain optimal length of a chain and thus efficiently balance the energy dissipation. We explained in more details about the reasons and criteria for chain reconstruction in subsection IV.C.

After fixing the chains, next target is to locate the lower-level chain leader at each chain. Unlike PEGASIS, where leaders are chosen randomly in every round, our protocol selects leaders for every chain based on the remaining energy in each sensor of the chain. In addition, we suggest not to change these

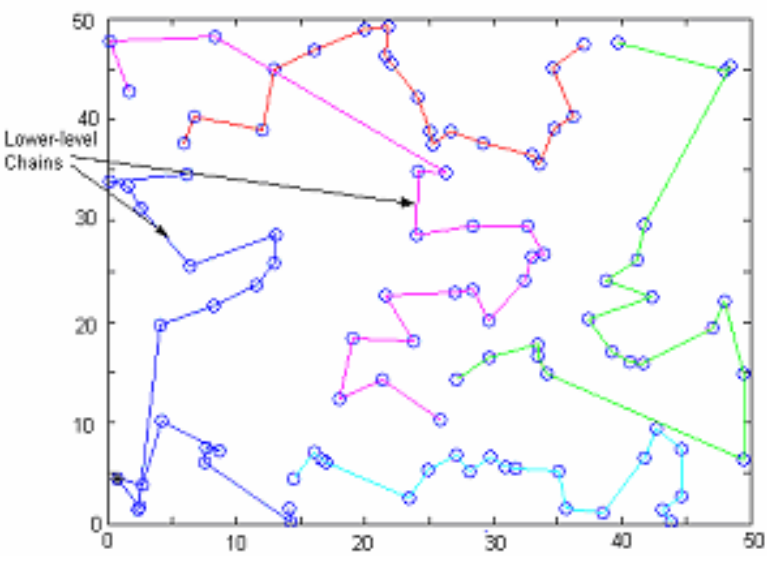

Figure 2: A snapshot of lower-level chain formation.

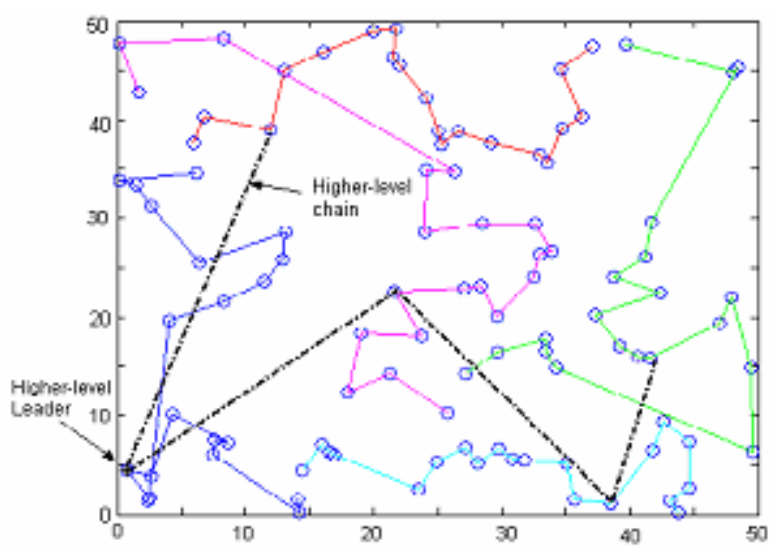

Figure 3: A snapshot of higher-level chain formation.

lower-level chain leaders at every round but after an optimal number of rounds. We tried to find out this optimal number of rounds $\mathrm{R}$, after that these local leaders are reselected based on criteria like energy expenditure and time required completing hundred rounds of data transfer cycle etc. The details of this simulation results are discussed in sub-section IV.D. The benefits of using a slight larger duration for selecting leaders rather than selecting leaders at every round are i) less communication overhead ii) reduced required time for leader selection at every round and iii) maximum utilization of higher level chain. Once these lower-level leaders are selected, a higher-level chain is formed again including all the lower-level leaders using the same greedy way as used in the formation of lower-level chain. After that a higherlevel leader is selected based on some criteria among those lower-level leaders that gathers all the information from other leaders and sends this information to the BS for further processing. For the 
higher-level leader selection the criteria our protocol considers are i) distance from BS ii) remaining energy of the node. In this way the protocol tries to ensure that nodes closer to BS take turn to transmit frequently than the nodes those are far from the BS. As nodes at far from BS station require more energy to the nodes those are nearer to the BS. We tried to evenly distribute the load of long distance transmission and our protocol can use the energy of the network optimally. Figure 2 shows a snapshot of lower-level chain formation in a 100 -node sensor field. All these nodes are grouped into five chains with 20 nodes in each, covering the whole geographical region. These small chains facilitate us to pull together information parallelly from the whole region. Figure 3 shows a snapshot of higher-level chain formation which includes all the lower-chain leaders and only one higher-level leader.

\section{B. Data Collection and Transmission Phase}

Data collection and transmission phases are combined in each round. During that data from all the nodes are collected and sent to the BS. Usually in sensor networking scenario, nodes sense the physical phenomenon of the environment such as temperature, heat, pressure etc. and after collecting raw data some kind of aggregation / data fusion takes place in order to minimize the length of data packet [18, 19]. Data aggregation can occur in two ways, firstly, at the node level that collects and senses the environment may aggregate its own information and secondly, while routing other node's information it can perform aggregation on its own data with the neighbor's data to provide a fixed length packet. After the lower-level chain and higher-level chain formation and the associated leader selection are accomplished, data collection and transmission phase come into action. But it is not obvious that chain formation phase always precede data collection phase, it precedes data collection phase whenever it is necessary to reconstruct new chains.

In our protocol the similar data transmission mechanism takes place both in the lower-level as well as in higher-level chains. This is adopted from that of PEGASIS [19]. Each chain leader is responsible for collecting data from its associated chain. Lower-level leaders collect information from lower-level chains and higher-level leader is the one responsible to collect data from the all lower-level leaders through higherlevel chain and transmit this information to the remote BS to complete a round. At the beginning of a round, each leader in a lower-level chain sends a token towards the one end indicating the beginning of data transmission phase. Node at one end of the chain sends its data toward the leader node through intermediate

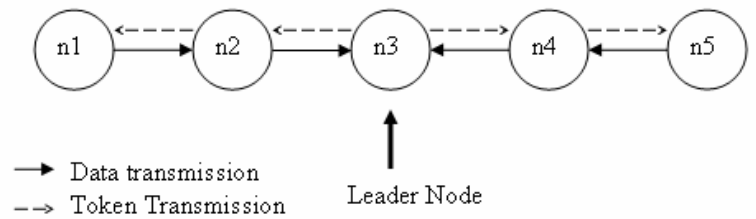

Figure 4: Data and token passing

nodes as shown in Figure 4. In this Figure leader n3 sends token toward node $\mathrm{n} 1$ through intermediate node $\mathrm{n} 2$. After getting this initiation message node $\mathrm{n} 1$ sends its data to node $\mathrm{n} 2$ which then fuses its own data with $\mathrm{n} 1$ 's data and send the final data packet to the leader node $n 3$. After receiving message from one end, $n 3$ sends token similarly toward node $\mathrm{n} 5$ and collect $\mathrm{n} 5$ and n4's data in the same way as described before. As the size of the token is small so cost associated to it is negligible. It is noticeable that data get fused at the time of traveling toward the leader node therefore leader only receives the aggregated information from its chain. As stated in the articles [11, 18, 19], this kind of data fusion can save a lot of energy which yields longer network lifetime. Therefore all the lower-level leaders collect data from their own chains using similar token and data passing approach parallelly. This parallel method of data collection results in reducing the delay in completing each round as compared to PEGASIS which utilizes a single chain. Now these lower-level leaders on the other hand form a higherlevel chain and among this higher-level chain only one node acts as higher-level leader. This higher-level leader now takes initiative to collect information from other lower-level leaders in a similar manner as used in lower-level chain. When this higher-level leader accumulates all leaders' data, it sends this data to the remote BS. It is worth mentioning that data undergoes further compression while travel along the higher-level chain toward the higher-level leader.

\section{SIMULATION AND ANALYSIS}

In order to approximate energy and time requirements in LEACH, PEGASIS and COSEN to complete a single round we consider the following assumptions: LEACH uses direct communications from cluster heads to the BS and $5 \%$ of the nodes act as cluster heads. Therefore, with around $5 \%$ of nodes acting as cluster heads in a 100-node network there are at least five long distance transmissions from five cluster heads to BS. Time required to complete a single round can be estimated in the following way: in an ideal case for LEACH, if $5 \%$ of the nodes are cluster heads in a 100-node network then there are approximately 20 nodes per cluster including the cluster-head. And with a TDMA schedule for 
Table 1. Comparative analysis among LEACH, PEGASIS and COSEN (single round for 100 -nodes)

\begin{tabular}{|l|l|l|l|}
\hline Parameters & LEACH & PEGASIS & COSEN \\
\hline Number of Long Distance Transmissions & 5 & 1 & 1 \\
\hline Unit Delay & 23 & 99 & 23 \\
\hline Others & $\begin{array}{l}\text { Dynamic cluster } \\
\text { setup overload }\end{array}$ & $\begin{array}{l}\text { Excessive } \\
\text { delay }\end{array}$ & $\begin{array}{l}\text { No cluster setup overload } \\
\text { Reasonable delay }\end{array}$ \\
\hline
\end{tabular}

19 nodes, requires approximately $19 t$ unit of time to collect data from all the nodes in a cluster where $t$ is the unit of time that is required for one node to transmit information to the cluster head. After that with a CSMA MAC any one among five cluster heads may have to wait at most $4 t$ unit of time before sending data to BS. In total $23 t$ unit of time may be required before sending the information from the last cluster head in any round. This time requirement may vary due to the randomness of the network and inherent problems related to the transmission in wireless medium but we consider here the ideal case for LEACH.

In case of PEGASIS, each node transmits to the next and receives from its previous nearest neighbor except the end two nodes in a chain. Only one leader is selected at each round to collect the aggregated data from other nodes and transmits this data to the BS. So long distance transmission reduces to possible minimum i.e. only one. But at the same time this single chain may introduce an excessive delay for distant nodes in the chain to transmit to the BS. If we assume it needs approximately same unit of delay $t$, to transmit from one node to the next node, then for an N-node network, if the leader is the end node in the chain, other end needs $(\mathrm{N}-1) t$ unit of delay to reach the leader node. Therefore, for a 100 -node network the delay yields $99 t$ units. Obviously this delay increases with the network size.

We propose to form several smaller chains with the deployed nodes each of them is of fixed length. All nodes are divided into N/CL chains where $\mathrm{N}$ is the total number of nodes and $\mathrm{CL}$ is the maximum number of nodes in a chain. As described in sub-section IV.B, in our experiment we use $\mathrm{CL}=20$. For a network of 100 nodes, we construct five chains each containing 20 nodes. Therefore, in extreme case, in order to reach the end node in a chain there are always a delay of (CL-1) $t$ i.e. $19 t$ units of time in each chain. This is the worst condition that may happen in our protocol. Even if we increase the network size but as the maximum number of nodes in a chain is fixed and that is $C L=20$, so the maximum possible delay within the lower-level chain is unchanged in our protocol. There are some additional delays in the higher-level chain. For a 100node network there are five leader nodes. Therefore extra (N/CL-1)t i.e. $4 t$ unit of delay may occur in the worst case before sending the signal to the BS. In total there may be (N/CL-1) $t+(\mathrm{CL}-1) t$ units i.e. $23 t$ units of delay for a 100-node network. Table-1 shows a comparison between LEACH, PEGASIS and COSEN in one round of data transmission for a 100-node network. It is clear from Table-1 that COSEN outperforms LEACH by avoiding the overload caused by dynamic cluster setup and minimizing the number of long distance transmissions. At the same time it causes much less delay to deliver information to the BS from distant nodes as compared to PEGASIS.

\section{A. Experimental Setup}

We developed our simulation program written in object oriented programming language $\mathrm{C}++$. Similar to LEACH and PEGASIS, we consider a network of 100 nodes and a fixed BS. The nodes are placed randomly in a place of 50 meter $\times 50$ meter and the BS is located at $(25,150)$. We use Cartesian coordinates to locate the sensors. We assume each sensor starts with one Joule of initial energy.

In practice it is difficult to model energy expenditure in radio wave propagation. Therefore in order to measure the energy expenditure in the network, we choose to use the same simplified radio model used in LEACH and PEGASIS. In LEACH they used the first order radio model and it is assumed that the sources of energy dissipation are the transmitter which dissipates energy to run radio electronics and power amplifier, and the receiver which dissipates energy to run the radio electronics. They approximate that the transmitter amplifier requires $E_{a m p}=100$ $\mathrm{pJ} / \mathrm{bit} / \mathrm{m}^{2}$ to amplify the signal at an acceptable signal to nose ratio (SNR). In addition energy required in running transmitter and receiver electronics are equal and given by $E_{t x-e l e c}=E_{r x-e l e c}=E_{\text {elec }}=50 \mathrm{~nJ} / \mathrm{bit}$. Moreover, the energy cost for data aggregation is considered as $5 \mathrm{~nJ} / \mathrm{bit} /$ message [18]. The bandwidth of the channel was set to $1 \mathrm{Mb} / \mathrm{s}$ [22]. In our experiments each data message is 2000 bits long and information processing time in a node is taken between 5 to 10 milliseconds [22]. The medium is assumed to be symmetric such that the energy required for transmitting a message 
from node A to B and from B to A are same at a fixed SNR. So we can say, for free space propagation loss, energy dissipation is certainly dominated by the long distance transmissions.

Thus the total transmission cost for a k-bit message is given by the Equation 1.

$$
E_{t x}(k, d)=E_{\text {elec }} * k+E_{\text {amp }} * k * d^{2}
$$

Here $\mathrm{d}$ is the distance between sender and receiver measured in meters. In case of receiving message, the energy consumption equation is given by Equation 2 .

$$
E_{r x}(k)=E_{\text {elec }} * k
$$

\section{B. Optimum Number of Nodes}

Optimum number of nodes, CL, is the number of nodes that should be included in each chain during the chain construction phase. We found from simulation result that if the number of nodes in a chain is fewer than this optimal number then both the required time and energy expenditure increase in the network. Also if the number of nodes is more than this value, energy requirements may decrease but time requirement increases. Therefore we tried to compromise both the energy expenditure and latency to find out a range that would be useful for our application. It seems that if the application requires less delay than we should choose lower number of nodes in a chain and if it requires low energy expenditure then number of nodes in a chain may be more. We consider several issues to choose the value of CL. At first, consider Figure 5. It shows the comparison for time required by our protocol for different CL values for 100 rounds. It is obvious from Figure 5 that if the CL value increases, required time also increases. The time differences are considerable enough. Figure 5 recommends that the value of $\mathrm{CL}$ should be in between 10 to 15 . Now consider the Figure 6, it depicts the energy required by the protocol for different values of CL. But it is apparent that the differences between energy requirements are little. From Figure 5 and Figure 6 we conclude that the value of CL could be in between 15 to 20 . In our work we consider $\mathrm{CL}=20$.

\section{Chain Reconstruction}

It is important to reconstruct the chains whenever a notable number of sensors die in the network. Otherwise, there may be possibilities that one chain contains higher number of sensors while other contains lower number of sensors. This may affect the performance of the protocol. It is notable in COSEN to maintain uniformity in number of sensors in all chains as only one sensor (i.e. the higher-level chain leader) is

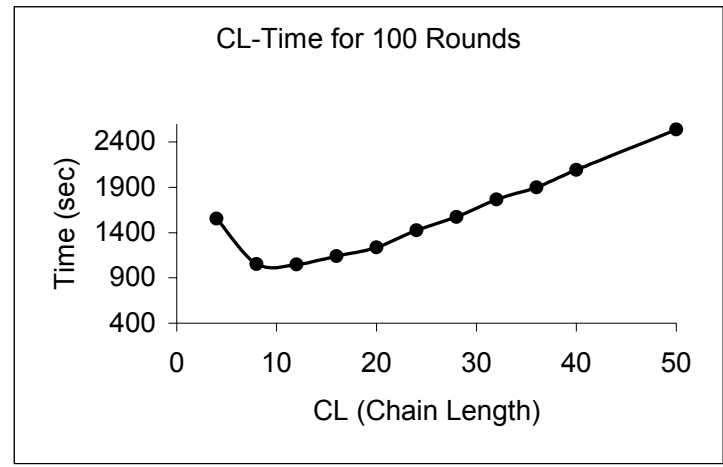

Figure 5: Time required vs. CL

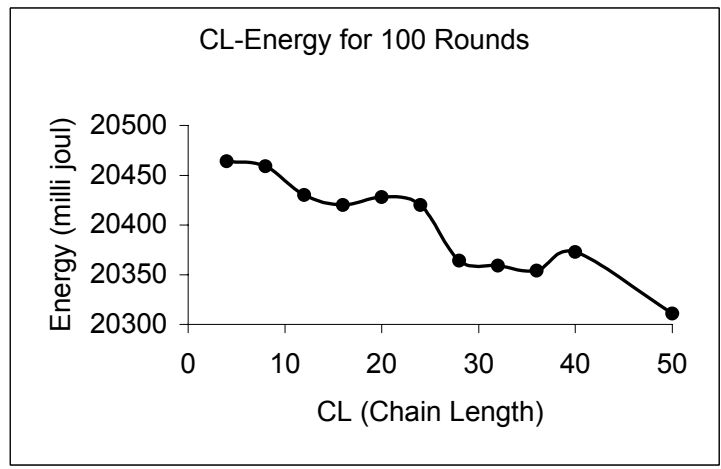

Figure 6: Energy required vs. CL

responsible to send the aggregated data to the $\mathrm{BS}$ and it has to wait for aggregated data from different lowerlevel chains. The uniformity of number of sensors in chains may also effect the energy dissipation and hence network lifetime too. If a chain consists of lower number of sensors, the probability of a sensor in that chain to be elected as local leader will be higher. Thus a short-chain is likely to loose sensors more often. Now, the question arises when we should reconstruct the chains in COSEN. The criterion we choose is the number of sensors died in the network. It is obvious that if we reconstruct the chains frequently, e.g. whenever only $4 \%-5 \%$ of the sensors die, it causes extra overhead. On the other hand, if we reconstruct chain belatedly, e.g. whenever $40 \%-50 \%$ of the sensors die, the uniformity among the chains may be destroyed. To find the optimal value, we reconstructed the chains varying the percentage of sensors' death in the network. In the simulation we reconstruct the chains when $4 \%, 8 \%, 12 \% \ldots$ etc. of sensors died in the network and observe the characteristics like total energy spent, lifetime of the network and time required to complete rounds. Figure $7(\mathrm{a}, \mathrm{b}, \mathrm{c})$ show the simulation results. Figure 7 (a) shows that energy consumption increases whenever chains are reconstructed less frequently. But the amount of energy 


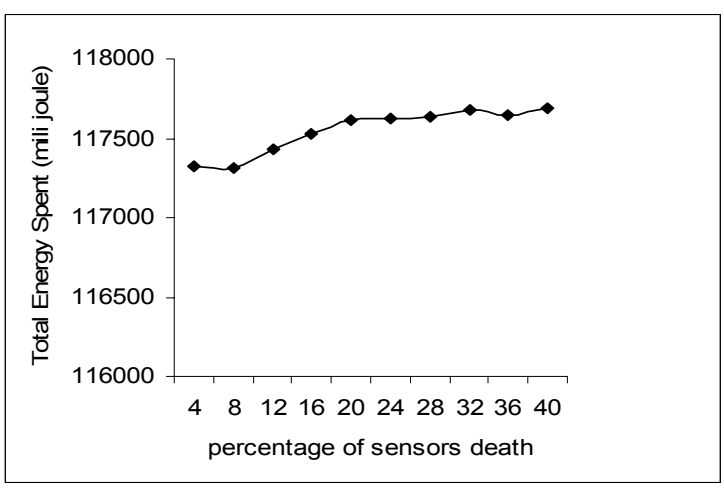

(a)

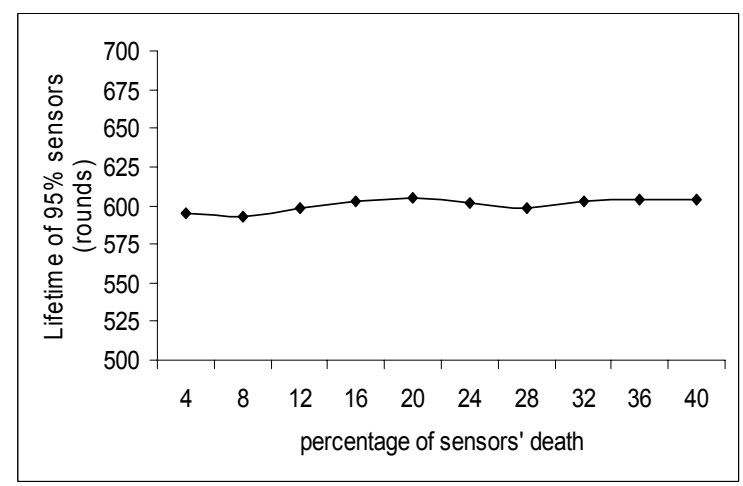

(b)

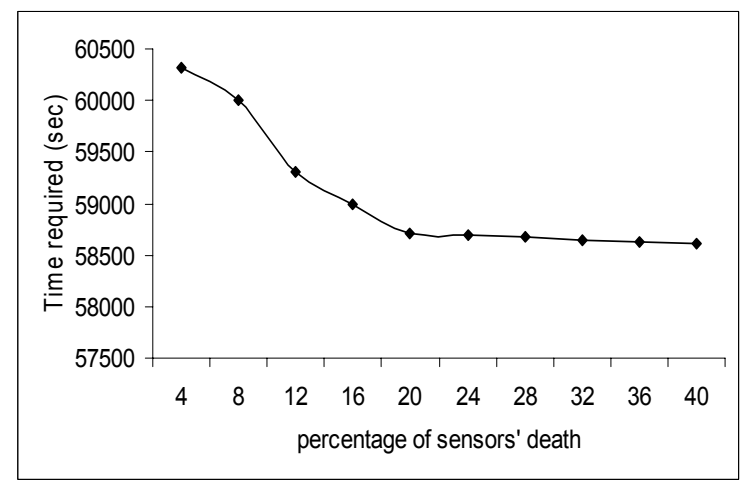

(c)

Figure 7: Simulation results regarding when to reconstruct chains in the network. (a) total energy spent (b) network lifetime and (c) time requirements vs. percentage of sensors' death

difference is not that huge. Figure 7(b) shows that the lifetime remains almost steady with a little pick around $20 \%$ sensor death. Figure 7(c) shows that time requirements decrease whenever chains are reconstructed less frequently. Time requirements sharply fall between $4 \%-20 \%$ of sensors' death and then remain almost the same. Thus we conclude to reconstruct chains whenever every $20 \%$ of the sensors

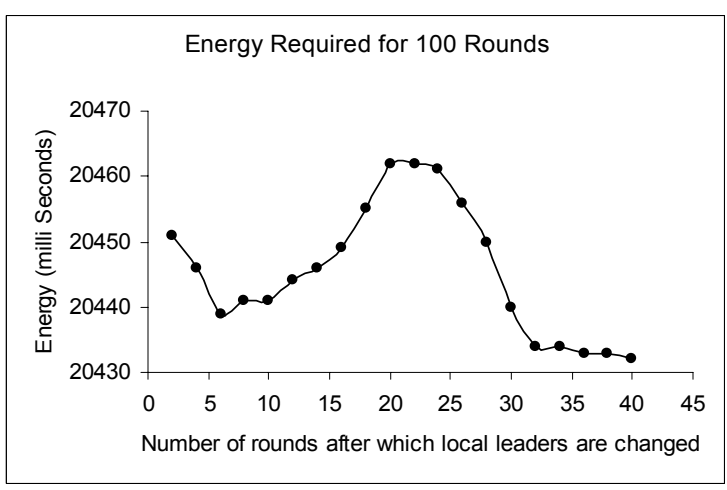

Figure 8: Energy dissipation vs. $\mathbf{R}$

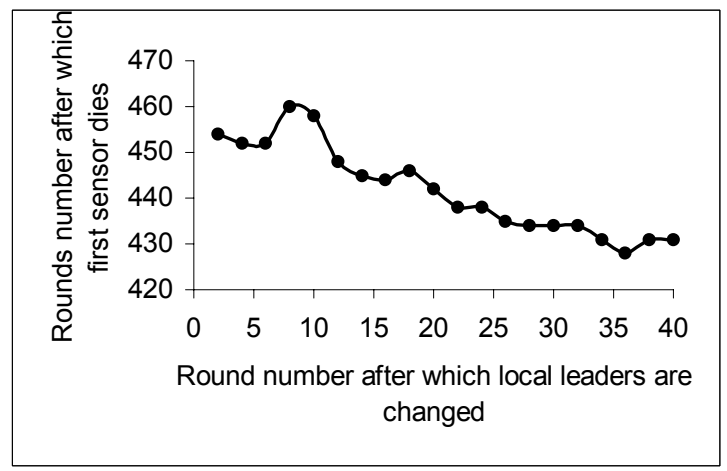

Figure 9: Rounds when 1st node dies vs. no. of rounds after the local leaders are changed

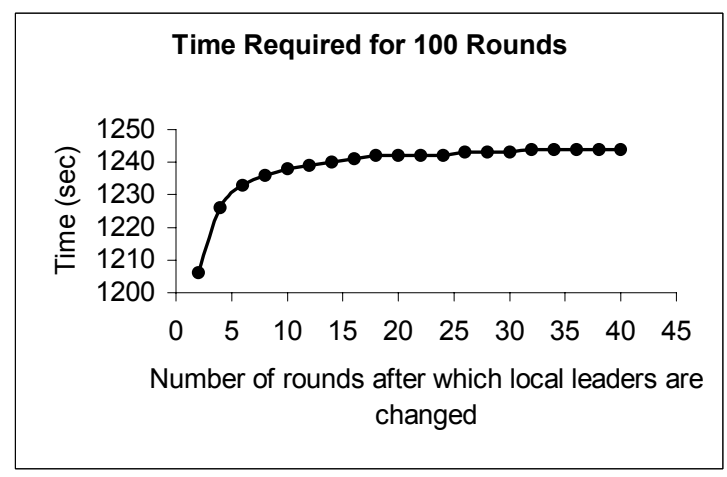

Figure 10: Time required vs. $\mathbf{R}$

of the network expire. Now the question arises how a leader knows that $20 \%$ of the nodes in its chain are dead. As described in sub-section III.B and depicted in figure 4, when data is fused in every sensor of a chain, each sensor can adds its tag with the data packet. For example, in figure 4 , normally $\mathrm{n} 1$ sends data to $\mathrm{n} 2 ; \mathrm{n} 5$ sends data to $\mathrm{n} 4$ etc. but if $\mathrm{n} 2$ is dead, $\mathrm{n} 1$ sends data directly to $\mathrm{n} 3$, and thus $\mathrm{n} 3$ can know that $\mathrm{n} 2$ is dead. In this way every lower chain leader come to know how many of its members are dead. In the similar fashion when the higher-level leader gets data from all lowerlevel leaders, it knows how many sensors already have died then it can send instruction accordingly to all. 


\section{Optimum Number of Rounds}

The lower-level leaders should be changed after some period of time to distribute the energy load. Next we have to decide the optimal number of rounds $\mathrm{R}$, after that the lower-level leaders need to be changed. If the lower-level leaders are changed at every round it causes extra energy expenditure for negotiations as well as causes delay. Also the higher-level chain would be utilized fully if we change these leaders after some number of rounds. On the contrary, if we do not change these leaders for long time they will quickly drain out energy because of excessive long transmissions. Therefore we consider to change the lower-level leaders based on the criteria like total energy dissipation in the network, maximum number of round when first node dies and delay introduced in the network against the different values of number of rounds. Simulation results by changing the rounds after which local leaders are changed are given in Figures 8, 9 and 10. From these figures we find that the value of $\mathrm{R}$ can be used for optimal result is between CL/4 to $\mathrm{CL} / 2$. In our case we considered this value as $\mathrm{CL} / 4$.

\section{RESULT}

We executed multiple runs of the simulation for each protocol and took the average value from those runs. The metrics we considered to measure performance of each protocol are i) overall energy expenditure in the network ii) number of rounds when the first sensor dies and iii) time to complete a round as well as hundred rounds.

As we compare the performance of our algorithm with that of PEGASIS, we consider the same number of nodes and size of sensor field for sensor deployment. We restricted the performance comparison between COSEN and PEGASIS because in [19] we find that, for the case of energy consumption, PEGASIS performs better than LEACH by about $100 \%$ to $300 \%$

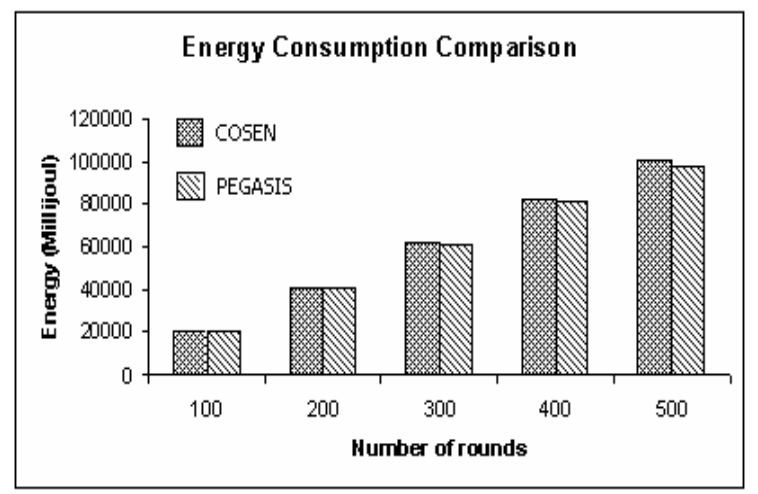

(a)

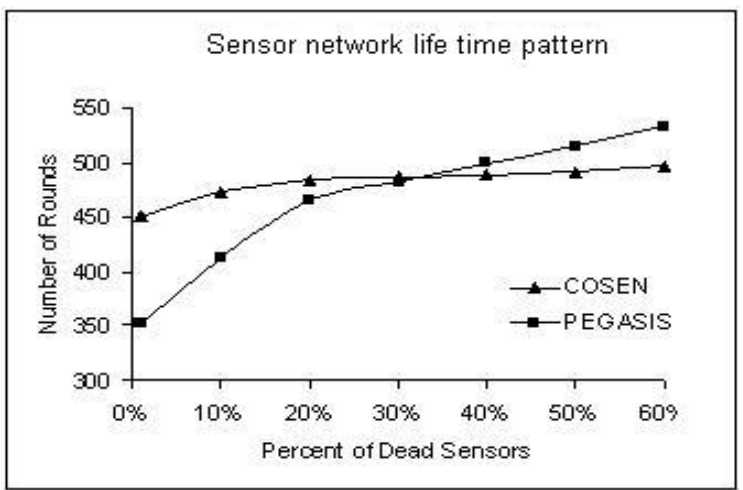

(b)

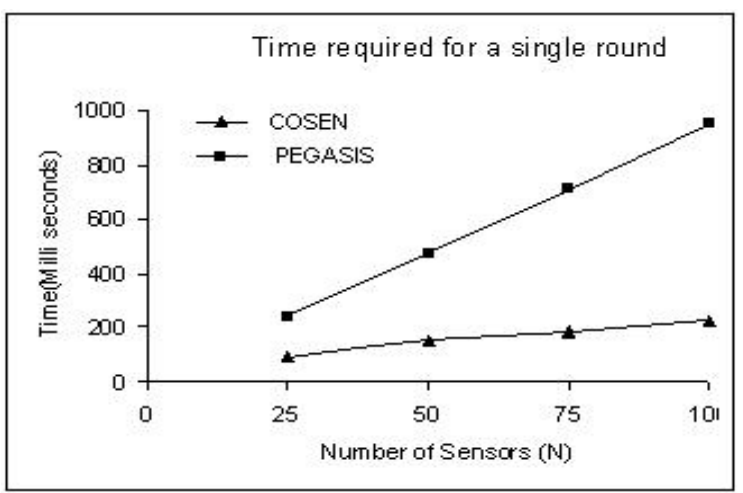

(c)

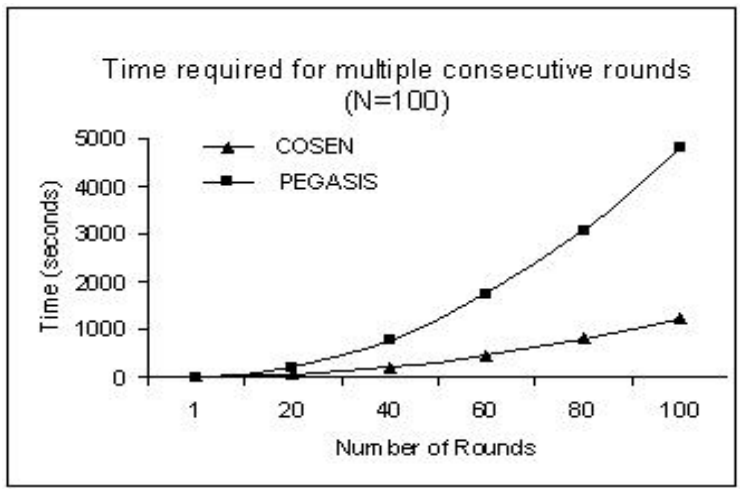

(d)

Figure 11: Simulation results regarding (a) energy dissipation comparison (b) lifetime of COSEN and PEGASIS (c) time requirement for single round and (d) time requirements for multiple consecutive rounds.

when $1 \%, 20 \%, 50 \%$, and $100 \%$ of nodes die for different network sizes and topologies.

Figure 11(a) demonstrates the comparative energy consumption by COSEN and PEGASIS. It is obvious from the figure that energy requirements for two protocols are almost same. For example, in case of total aggregated energy consumption for all sensors in the network, after 100 rounds COSEN requires only 0.218 joules of energy additional than that of 
PEGASIS, after 500 rounds COSEN requires only 2.833 joules of energy additional than that of PEGASIS etc. But the significant point for COSEN is that, it spends the energy in a totally distributed way such that the network can operate higher number of rounds before the first sensor dies. The lifetime pattern of COSEN is depicted in Figure 11(b). Here we can see that the first node dies for PEGASIS at 350 rounds, but the first node dies at around 450 rounds for COSEN. As PEGASIS is superior to LEACH for energy consumption by minimum $100 \%$, it can be derived that COSEN also outperforms LEACH in case of energy consumption.

The definitive improvement of COSEN over PEGASIS is that, the latency in data delivery is greatly reduced. It is noticed that latency of PEGASIS increases exponentially when number of rounds in the sensor network increases. Figure 11(c) shows that where, for 100 rounds, PEGASIS requires around 5000 seconds, COSEN requires only one-fifth of that time.

\section{CONCLUSION AND FUTURE WORK}

In this paper, we propose a chain based hierarchical routing approach for sensor network in an energy and time constraint environment. For simplicity and ease to understand we limited our hierarchy level within two layers. But for a sensor network with huge number of nodes this very protocol can be extended to multiple hierarchical layers, remaining the protocol constitution unchanged. The protocol exhibits outstanding results in efficiency in both energy consumption to support the network for a longer time and also the time requirement to meet the network real-time needs. Simulation results show that our protocol exhibits an excellent time-energy compromise as compared to that of both LEACH and PEGASIS. Moreover, we find in the simulation that our protocol performs higher number of rounds than that of PEGASIS before the first sensor dies. Future work will involve a more detailed study of the performance in the face of failures, as well as comparisons with additional algorithms. Moreover, in future we would like to enhance the protocol to fit with moving-sensors environment.

\section{REFERENCES}

[1] R. Szewczyk, J. Polastre, A. Mainwaring, and D. Culler, "Lessons from a sensor network expedition," Proc. 1st European Workshop Wireless Sensor Networks (EWSN'04), Berlin, Jan. 2004, pp. 1-16.

[2] S. Tilak, N. B. Abu-Ghazaleh, and W. Heinzelman, "A taxonomy of wireless micro-sensor network models," SIGMOBILE Mob. Comput. Commun. Rev., vol. 6, no. 2, 2002, pp. 28-36.
[3] Q. Jiang and D. Manivannan, "Routing protocols for sensor networks," Proc.1st IEEE Consumer Comm. \& Net. Conf. (CCNC), Jan.2004, pp. 93-98.

[4] I. F. Akyildiz et al., "Wireless Sensor Networks: A Survey," Elsevier Sci. B. V. Comp. Networks, vol. 38, no. 4, Mar. 2002, pp. 393-422.

[5] D. Estrin, L. Girod, G. Pottie, and M Srivastava, "Instrumenting the world with wireless sensor networks," Proc. ICASSP, Salt Lake City, Utah, May 2001.

[6] A. Schmidt, and K.V. Laerhoven, "How to build smart applications?," IEEE Personal Communications, Aug. 2001, pp. 66-71.

[7] L.P. Clare, G.J. Pottie, and J.R. Agre, "Self-Organizing Distributed Sensor Networks," SPIE Conference on Unattended Ground Sensor Technologies and Applications, Apr. 1999, pp. 229-237

[8] M. J. Dong, K. G. Yung, and W. J. Kaiser, "Low Power Signal Processing Architectures for Network Micro sensors," Proc. 1997 International Symposium on Low Power Electronics and Design, Aug. 1997, pp. 173-177.

[9] K. Shohrabi and G. Pottie, "Performance of a Novel SelfOrganization Protocol for Wireless Ad-Hoc Sensor Networks," Proc. of the IEEE 50th Vehicular Technology Conference, 1999, pp. 1222-1226.

[10] J. N. Al-Karaki and A. E. Kamal, "Routing Techniques in Wireless Sensor Networks: A Survey," IEEE Wireless Communications, vol. 11, no. 6, Dec 2004.

[11] W. B. Heinzelman, A. Chandrakasan, and H. Balakrishanan, "An Application-Specific Protocol Architecture for Wireless Micro sensor Networks," IEEE Trans. Wireless Commun., vol. 1, no. 4, Oct. 2002, pp. 660670.

[12] N. Tabassum, Q.E.K. Mamun and Y. Urano," COSEN: A Chain Oriented Sensor Network for Efficient Data Collection," Proc. of Third International Conference on Information Technology : New Generations, Las Vegas, Nevada, USA, Apr 10-12, 2006.

[13] N. Tabassum. Q.E.K. Mamun, and Y. Urano, "An Energy-Aware Protocol for Periodical Data Collection in Wireless Sensor Networks," Proc. of First IEEE Intl. Conf on Wireless Broadband and Ultra Wideband Communications, Sydney, Australia, Mar 13-16, 2006.

[14] K. Akkaya and M. Younis, "A Survey of Routing Protocols in Wireless Sensor Networks," Elsevier Ad Hoc Network Journal, Vol. 3/3, 2005, pp. 325-349.

[15] L. Klein, Sensor and Data Fusion Concepts and Applications. SPIE Optical Engr Press, WA, 1993.

[16] D. Niculescu, "Communication paradigms for sensor networks," Communications Magazine, IEEE, Vol.43, No. 3. Mar. 2005, pp. 116-122.

[17] S.D. Muruganathan, D.C.F. Ma, R.I. Bhasin, and A.O. Fapojuwo, "A centralized energy-efficient routing protocol for wireless sensor networks," IEEE Radio Communications Magazine, Mar 2005, pp. 8-13. 
[18] W. R. Heinzelman, A. Chandrakasan, and H. Balakrishnan, "Energy-efficient Communication Protocols for Wireless Micro sensor Networks," Proc. of the 33rd Hawaii International Conference on System Sciences, Jan. 2000 .

[19] S. Lindsay and C. Raghavendra, "PEGASIS: PowerEfficient Gathering in Sensor Information Systems," in international Conf. on Communications, 2001.

[20] A. Manjeshwar and D.P. Agrawal, "TEEN: A Routing Protocol for Enhanced Efficiency in Wireless Sensor Networks", 1st Intl. Workshop on Parallel and Distributed Computing, Apr 2001.

[21] N. Bulusu, J. Heidemann, and D. Estrin, "GPS-less Low Cost Out Door Localization for Very Small Devices," IEEE Personal Communication, Oct 2000, pp. 28-34.

[22] J. Kulik, W. R. Heinzelman, and H. Balakrishnan, "Negotiation-based protocols for disseminating information in wireless sensor networks", Wireless Networks, vol. 8, Mar. 2002, pp. 169-185.

\section{BIOGRAPHIES}

Nahdia Tabassum received her B.Sc. and M.Sc. degree in Applied Physics and Electronics from Rajshahi University in 1997 and 2000 respectively. She worked in Aptech, PGD in IT, Rajshahi University and then in Dept. of ICT, Islamic University, Bangladesh as a teaching member from 2001 to 2004. Afterwards she left for Japan in 2004 for higher studies. Currently she is pursuing MS in GITS, Waseda University, Japan. Her research topic is energy and delay sensitive routing for wireless sensor networks. She is a member of BCS and student member of IEICE.
Quazi Ehsanul Kabir Mamun earned his B.Sc. degree in Computer Science and Engineering from Bangladesh University of Engineering and Technology in 2001. Then he joined The University of Asia Pacific, Dhaka as Lecturer of CSE department and served there from 2001 to 2004. Currently he is enrolled in MS program in GITS of Waseda University, Japan. His research interests include wireless sensor networks and distributed systems. He is a student member of IEICE.

AKM Ahsanul Haque is a Lecturer at the ECE Discipline of Khulna University, Bangladesh. He received his B.Sc. (Honors) in Electronics from University of Delhi in 1998 and M.Sc. degrees in Electronics from Jamia Millia Islamia University, New Delhi, India in 2000. His research interests are networking protocol for wireless and ad hoc networks, in particular sensor networks. He is a member of BCS.

Dr. Yoshiyori Urano is a professor, a Dean of the Graduate School of Global Information and Telecommunication studies (GITS), Waseda University, Japan. His research interests cover technologies for the construction of next-generation multimedia computer networks and the development of their applications. These applications include multimedia distributed processing platform, distributed and collaborative work, and network applications such as distance education, telemedicine and assistive technology. He currently serves as an expert on the Telecommunications Technology Council, Ministry of Internal Affairs and Communications, Japan and as Vice Chair of WG3, Asia Info-Communications Council. 